\section{Subphrenic hematoma mimicking malpositioned gallbladder during endoscopic retrograde cholangiopancreatography}

A 60-year-old woman with recurrent upper abdominal pain of 10 days' duration visited our hospital. Abdominal computed tomography demonstrated a stone in the common bile duct (CBD), and endoscopic retrograde cholangiopancreatography (ERCP) was performed to remove it. The patient's hematological and clotting parameters were normal.

Deep biliary cannulation with a 0.035 inch straight-tip guidewire (Jagwire; Boston Scientific, Natick, Massachusetts, USA) was attempted. The CBD stone was extracted successfully with endoscopic sphincterotomy and a disposable extraction basket (Endo-Flex, Voerde, Germany). However, cholangiography showed an

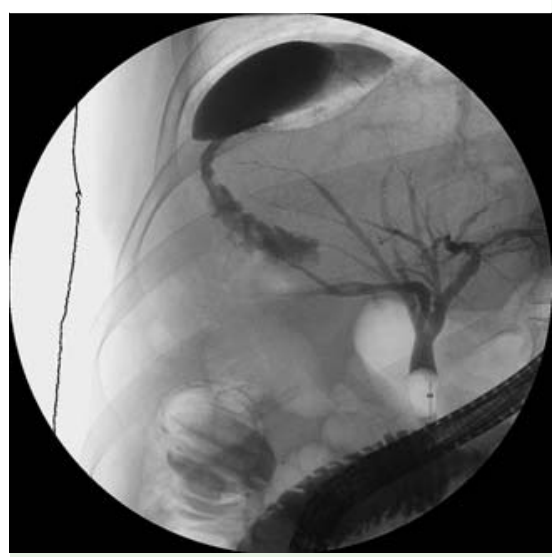

Fig. 1 Occlusive cholangiography during endoscopic retrograde cholangiopancreatography in a 60-year-old woman with recurrent upper abdominal pain of 10 days' duration showing leakage of contrast from the right intrahepatic duct to the subphrenic area. iation of the gallbladder. catheter. ovoid collection of contrast at the upper pole of the liver. In particular, occlusive cholangiography demonstrated leakage of contrast from the right intrahepatic duct to the subphrenic area ( $\bullet$ Fig. 1). We assumed this lesion to be a positional var-

On the following day, the patient continued to experience vague right upper quadrant pain without tenderness or signs of peritonitis. Follow-up abdominal computed tomography demonstrated a $10 \times 6-\mathrm{cm}$ air-filled hematoma mixed with contrast in the subphrenic area, complicated by a pleural effusion ( $\bullet$ Fig. 2). On a coronal computed tomographic scan, we could distinguish the gallbladder from the subphrenic hematoma ( $\bullet$ Fig. 3 ). The patient was hemodynamically stable, and the hematoma was successfully treated by percutaneous drainage with a pigtail

Although several cases of subcapsular hematoma have been diagnosed by computed tomography several days after ERCP, $[1,2]$ this is the first report in the literature of a subphrenic hematoma visualized by cholangiography during ERCP. Guidewire trauma into the liver parenchyma, by deep cannulation into the intrahepatic bile duct and the forceful injection of contrast during occlusive cholangiography are thought to be contributing factors. The iatrogenic formation of a subphrenic hematoma during ERCP can be prevented by cautious manipulation of the guidewire and the avoidance of excessive pressure during occlusive cholangiography.

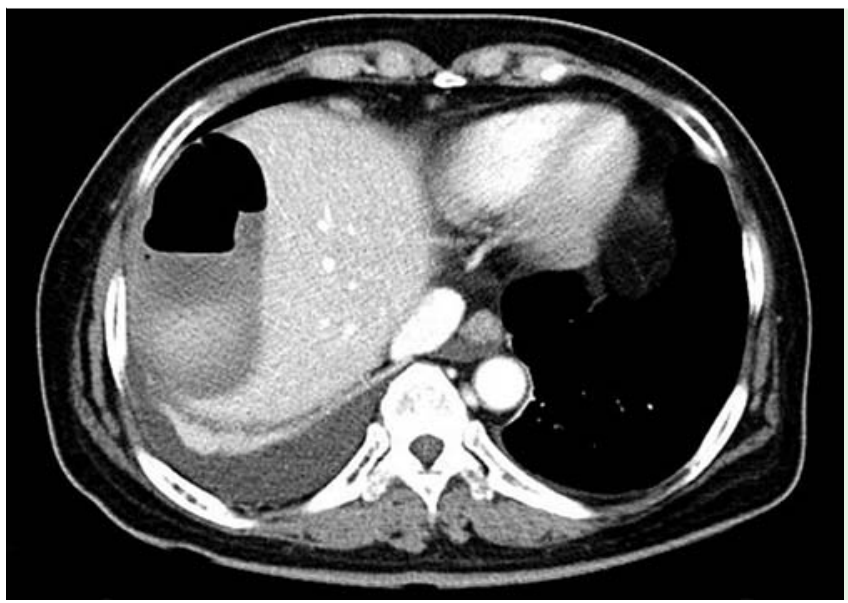

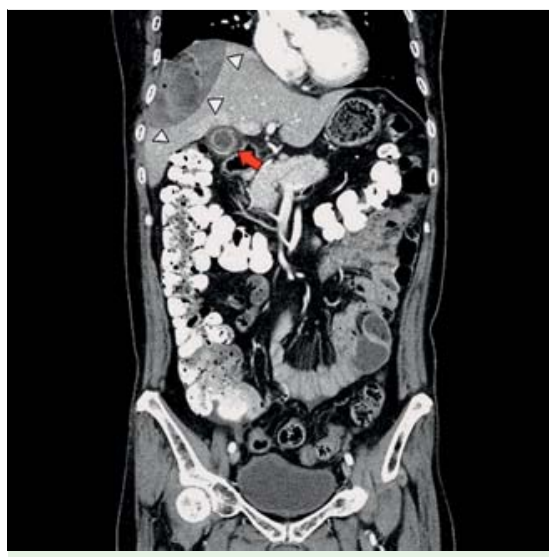

Fig.3 Coronal abdominal computed tomographic scan showing the gallbladder (red arrow) and subphrenic hematoma (arrowheads).

\section{Endoscopy_UCTN_Code_CPL_1AK_2AC}

Competing interests: None

\section{Yo Han Jeong, Tae Nyeun Kim, Kook Hyun Kim}

Department of Internal Medicine,

Yeungnam University College of Medicine, Daegu, Korea

\section{References}

1 Manikam J, Kaur S, Mahadeva S et al. Subcapsular hematoma of the liver following endoscopic retrograde cholangiography: a known but rare complication. Dig Endosc 2011; 23: 209-210

2 Orellana F, Irarrazaval J, Galindo J et al. Subcapsular hepatic hematoma post ERCP: a rare or an underdiagnosed complication? Endoscopy 2012; 44 (Suppl. 02): E108E109

3 McArthur KS, Mills PR. Subcapsular hepatic hematoma after ERCP. Gastrointest Endosc 2008; 67: 379-380

Bibliography

DOI http://dx.doi.org/

10.1055/s-0034-1393043

Endoscopy 2015; 47: E521

(c) Georg Thieme Verlag KG

Stuttgart · New York

ISSN 0013-726X

\section{Corresponding author \\ Kook Hyun Kim, MD}

Department of Internal Medicine

Yeungnam University College of Medicine

170 Hyeonchungro

Nam-gu

Daegu 705-717

Korea

Fax: +82-53-654-8386

kimkh@yu.ac.kr 